\title{
Metafluid with anisotropic dynamic mass
}

\author{
L.N. Gumen \\ Universidad Popular Autónoma del Estado de Puebla, 21 Sur, \#110, 72160, Mexico
}

J. Arriaga

Instituto de Fisica, Universidad Autónoma de Puebla, Apartado Postal J-48, Puebla 72570, Mexico

\author{
A.A. Krokhin \\ Department of Physics, University of North Texas, 1155 Union Circle \# 311427, Denton, TX 76203, USA \\ E-mail: arkady@unt.edu
}

Received June 24, 2011

\begin{abstract}
We show that a fluid filling the space between metallic cylinders arranged in a two-dimensional lattice exhibits anisotropic dynamic mass for sound waves propagating through the lattice, if its unit cell is anisotropic. Using the plane-waves expansion method we derive (in the long wavelength limit) a formula for the effective mass tensor of the metafluid. The proposed formula is very general - it is valid for arbitrary Bravais lattices and arbitrary filling fractions of the cylinders. We apply our method to a periodic structure with very high anisotropy, when other known methods fail. In particular, we calculate the effective mass tensor for sound waves in air with embedded lattice of aluminum cylinders having rectangular cross sections, and obtain excellent agreement with experiment. The proposed method of calculation may find numerous applications for tailoring of metafluids with prescribed anisotropy.
\end{abstract}

PACS: 62.65.+k Acoustical properties of solids; 43.20.+g General linear acoustics.

Keywords: sound waves, phononic crystal, homogenization, anisotropy.

\section{Introduction}

Anisotropy is a property which is usually associated with crystal solids. Fluids and glasses are naturally isotropic in the absence of external fields. Anisotropy may, however, be artificially stimulated by embedding periodic structures in naturally isotropic fluids. Then these artificial structures - so called phononic crystals - may have very unusual properties, see, e.g. [1]. Within a narrow band of frequencies of sound the effective mass and the effective elastic modulus of specially designed phononic crystals may become anisotropic, take negative values, or acquire abnormally large imaginary part. Due to such "strange" properties that do not exist for natural materials these artificial structures are usually called metamaterials or metafluids [2-5]. Since metamaterial behavior is due to multiple scattering of waves and internal acoustic resonances within periodic medium, the corresponding metamaterial parameters like mass density, elastic bulk modulus, and index of refraction, are essentially dynamical (effective). Static properties of a metamaterial remain "normal", i.e., they do not exhibit any anomalies.

Acoustic metamaterials have been intensively studied in the last decade, mostly because of their useful applications in acousto-electronics and medical acoustics. In particular, an acoustic superlens with tunable focal length based on metamaterial has been demonstrated [6] and also the subwavelength resolution has become possible due to the ability of a metamaterial with negative index of refraction to catch the evanescent modes from an object [7]. Another application of metamaterials is related to the problem of invisible cloaking of objects. Cloaking device provides advanced stealth technology that will cause a solid object to be partially or completely undetectable for radars and sonars. Modern progress in this field is due to so-called transformation optics [8] and transformation acoustics [9]. These techniques use a coordinate transformation in a wave equation (written for dielectric or elastic medium) which minimizes the scat- 
tering cross-section for the cloaked object. The transformation optics is based on the invariance of 3D Maxwell's equations under coordinate transformations [10]. The invariance means that the form of the wave equation is conserved under the coordinate transformation but the dielectric and magnetic properties of the medium are renormalized within a finite size layer around the object. This renormalization requires coordinate-dependent and tensorial constitutive relations within the cloaking layer [8]. Such relations do not exist for natural optical materials, nevertheless they have been realized using artificial structures possessing the required inhomogeneity and anisotropy in the long wavelength limit $[11,12]$.

While the wave equation for light that follows from the Maxwell's equations in many senses is similar to the wave equation in elastic media, the latter does not possess the necessary invariance [13]. Absence of such invariance strongly limits the transformation-based applications in acoustics, in particular, realization of acoustic cloaking [9]. There is, however, one important special case with one-toone correspondence between the electromagnetic and the acoustic wave equation. This is the case when the properties of a medium are independent on one of the coordinates (e.g. on the coordinate $z$ ) and the wave equations can be written in two dimensions [14]. A possibility of acoustic cloaking of a long cylinder has been demonstrated in Ref. 15. It is worth mentioning that in this case the invisible cloak consists of a set of concentric layers of parallel cylinders, i.e., it can be easier realized in practice [16-18].

Acoustic cloak in two-dimensions requires a finitewidth layer of a fluid with anisotropic (and inhomogeneous) mass density, i.e., the mass density is not a scalar but a $2 \times 2$ tensor $\rho_{i k}$ with different radial and azimuthal components [15]. It was shown, using multiple-scattering theory, that an anisotropic 2D lattice of rigid cylinders embedded in a fluid may provide anisotropy which is necessary for acoustic cloaking [17]. A different way to produce anisotropic fluid based on radially periodic corrugated acoustic cavities has been recently proposed in $[19,20]$.

Here we propose an analytic approach for calculation of the effective mass density tensor for a phononic crystal of solid cylinders embedded in a fluid. The method is based on the plane-wave expansion of the wave equation in the long wavelength limit (homogenization). As an example we consider anisotropic lattice of steel cylinders in air and show that our results for the components of the mass density tensor are in complete agreement with the recent numerical and experimental results obtained for the same structure $[21,22]$.

\section{Effective medium parameters for periodic arrangement of solid cylinders in a fluid}

We consider a 2D periodic structure of solid cylinders with arbitrary shaped cross-sections and their axes being parallel to axis $z$. The cylinders are embedded in a nonviscous fluid. Since a fluid supports propagation of only longitudinal waves and the solid cylinders are assumed to be pairwise disjoint, i.e., not touching one another (cermet geometry), the transverse waves are suppressed. Thus, the propagating mode is longitudinal and its phase velocity is expressed as follows:

$$
c(\mathbf{n})=\sqrt{\frac{B_{\mathrm{eff}}}{\rho_{\mathrm{eff}}}} .
$$

Here $B_{\text {eff }}$ and $\rho_{\text {eff }}$ are the effective bulk modulus and the effective mass density of the periodic composite medium. In general case a periodic medium is dispersive, i.e., the speed of sound depends on the Bloch vector $\mathbf{k}$. Here we consider the long wavelength limit, $k a \ll 1$, when the details of the periodic structure with typical size of the unit cell $a$ are not well resolved at the wavelength $2 \pi / k$. Due to lack of resolution (that is equivalent to lack of diffraction) the lowest band of a phononic crystal is always linear, $\omega=k c$, i.e., the speed of sound is a well-defined quantity in the long wavelength limit,

$$
c(\mathbf{n})=\lim _{k \rightarrow 0} \frac{\omega}{k} .
$$

The lack of diffraction does not mean that the microstructure of the medium is completely "ignored" by the propagating wave. One of the manifestations of this fact is the dependence of the speed of sound in Eq. (1) on the direction of propagation in the plane of periodicity, $\mathbf{n}=\mathbf{k} / k$. This dependence is the source of anisotropy in the mass density.

It has been known since the fundamental study of Wood [23] that the averaging of the bulk modulus and mass density over the wavelength does not necessary lead to the corresponding volume averages. Moreover, the result of averaging depends on the polarization of the propagating elastic wave. For longitudinal sound the reciprocal of the bulk modulus is averaged

$$
\frac{1}{B_{\text {eff }}}=\frac{f}{B_{c}}+\frac{1-f}{B_{f}},
$$

where $B_{c}$ and $B_{f}$ are the elastic moduli of the cylinders and fluid, respectively, and $f$ is the filling fraction occupied by the cylinders. Since $B_{\text {eff }}$ is a scalar which does not absorb any feature of the composite but $f$, all the details of the periodic microstructure are attributed to the effective mass density. The latter was first calculated by Berryman [24] for the case of isotropic composite (square lattice of circular cylinders). He used the approach similar to the Maxwell-Garnet approximation known for the dielectric composites [25], and for the effective mass of a fluid with embedded circular cylinders arranged in a square lattice obtained the following formula:

$$
\frac{\rho_{\mathrm{eff}}-\rho_{f}}{\rho_{\mathrm{eff}}+\rho_{f}}=f \frac{\rho_{c}-\rho_{f}}{\rho_{c}+\rho_{f}} .
$$


This old result has been recently justified by the multiple-scattering theory [26]. Although Eq. (4) is in a good agreement with the experiment [27], it is formally valid for small values of the filling fraction, $f \ll 1$ (or $1-f \ll 1$ ). It is also not applicable for the non-square lattices and for the cylinders with non-circular cross-section.

To obtain more general result which is valid for any Bravais lattice and any contrast between the acoustic impedances of the constituents, we use the method of plane waves. The long wavelength limit for the speed of sound in 3D periodic fluid-fluid system (e.g. air bubbles in water) was calculated in Ref. 28. This result is equally valid for 2D phononic crystal of solid cylinders embedded in fluid, since the transverse mode is suppressed. The speed of sound [28] can be written in the form of Eq. (2) with effective elastic modulus given by Eq. (3) and the effective mass density $\rho_{\text {eff }}(\mathbf{n})$ which contains the information on the microstructure of the phononic crystal

$$
\begin{aligned}
& \frac{1}{\rho_{\mathrm{eff}}(\mathbf{n})}=\frac{f}{\rho_{c}}+\frac{1-f}{\rho_{f}}- \\
& -\sum_{\mathbf{G}, \mathbf{G}^{\prime} \neq 0}(\mathbf{n} \cdot \mathbf{G})\left(\mathbf{n} \cdot \mathbf{G}^{\prime}\right) v(\mathbf{G}) v\left(-\mathbf{G}^{\prime}\right)\left[\mathbf{G} \cdot \mathbf{G}^{\prime} v\left(\mathbf{G}-\mathbf{G}^{\prime}\right)\right]^{-1} .
\end{aligned}
$$

Here $\mathbf{G}$ and $\mathbf{G}^{\prime}$ are the reciprocal lattice vectors of $2 \mathrm{D}$ periodic structure, $[\ldots]^{-1}$ implies inversion of the matrix in the brackets, and $v(\mathbf{G})$ are the Fourier components of the periodic function $1 / \rho(\mathbf{r})$

$$
\frac{1}{\rho(\mathbf{r})}=\sum_{\mathbf{G}} v(\mathbf{G}) \exp (i \mathbf{G} \cdot \mathbf{r}) \text {. }
$$

Equation (5) is exact, it takes into account all the dynamical and geometrical details of the periodic structure which affect propagation of sound in the long wavelength limit. In practice, the inversion of the matrix and sum over $\mathbf{G}$ and $\mathbf{G}^{\prime}$ require numerical calculation with finite number of reciprocal lattice vectors, i.e., with finite number of plane waves. It was shown that the sums over $\mathbf{G}$ and $\mathbf{G}^{\prime}$ converge rapidly with number of the plane waves [29], therefore numerical calculation of the sum is not a time consuming procedure at all.

For unit cells possessing third (or higher) rotational axis of symmetry the dependence on the unit vector $\mathbf{n}$ in the right-hand-side disappears, i.e., in the long wavelength limit these periodic structures are isotropic. Otherwise, Eq. (5) gives an ellipse for the effective mass $\rho_{\text {eff }}(\mathbf{n})$ in the $x y$ plane. In particular, for 2D lattice with rectangular symmetry the inverse dynamical mass density along $x$ - and $y$-direction can be written as follows:

$\frac{1}{\rho_{x}}=\frac{f}{\rho_{c}}+\frac{1-f}{\rho_{f}}-\sum_{\mathbf{G}, \mathbf{G}^{\prime} \neq 0} G_{x} G_{x}^{\prime} v(\mathbf{G}) v\left(-\mathbf{G}^{\prime}\right)\left[\mathbf{G} \cdot \mathbf{G}^{\prime} v\left(\mathbf{G}-\mathbf{G}^{\prime}\right)\right]^{-1}$,

$$
\frac{1}{\rho_{y}}=\frac{f}{\rho_{c}}+\frac{1-f}{\rho_{f}}-\sum_{\mathbf{G}, \mathbf{G}^{\prime} \neq 0} G_{y} G_{y}^{\prime} v(\mathbf{G}) v\left(-\mathbf{G}^{\prime}\right)\left[\mathbf{G} \cdot \mathbf{G}^{\prime} v\left(\mathbf{G}-\mathbf{G}^{\prime}\right)\right]^{-1} .
$$

For a rectangular lattice with periods $a_{x}$ and $a_{y}$ along axes $x$ and $y$, respectively, the reciprocal lattice vectors are $G_{x}=2 \pi m / a_{x}$ and $G_{y}=2 \pi n / a_{y}$, where $m$ and $n$ are integers.

\section{Numerical results and discussion}

We calculate the dynamic mass density for a phononic crystal of air background and solid cylindrical inserts. The background air has static mass density $\rho_{f}=1.29 \mathrm{~kg} / \mathrm{m}^{3}$ and the bulk modulus $B_{f}=141 \mathrm{kPa}$. The inserts are aluminum cylinders with $\rho_{c}=2712.63 \quad \mathrm{~kg} / \mathrm{m}^{3}$ and $B_{c}=68.9 \mathrm{Gpa}$ arranged in a square lattice with a period $a=0.953 \mathrm{~cm}$. The cylinders have a rectangular cross section with sides $b_{x}$ and $b_{y}$. We calculate the effective mass density as a function of filling fraction $f=b_{x} b_{y} / a^{2}$, keeping the same aspect ratio $b_{y} / b_{x}=5$ as was used in [21] and [22].

The Fourier components $v(\mathbf{G})$ of the inverse density $\rho(\mathbf{r})$ for this structure are

$$
\begin{gathered}
v(\mathbf{G})=v_{m n}=\frac{1}{\pi^{2} m n}\left(\frac{1}{\rho_{c}}-\frac{1}{\rho_{f}}\right) \sin \left(\frac{\pi b_{x} m}{a}\right) \sin \left(\frac{\pi b_{y} n}{a}\right), \\
m^{2}+n^{2} \neq 0 \\
v(\mathbf{G}=0)=\frac{f}{\rho_{c}}+\frac{1-f}{\rho_{f}} .
\end{gathered}
$$

The matrix $A\left(\mathbf{G}, \mathbf{G}^{\prime}\right)=\mathbf{G} \cdot \mathbf{G}^{\prime} v\left(\mathbf{G}-\mathbf{G}^{\prime}\right)$ to be inverted in Eqs. (7) and (8) has the following diagonal

$$
\begin{aligned}
& A(\mathbf{G}, \mathbf{G})=A_{m n, m n}=G^{2} v(\mathbf{G}=0)= \\
& =\frac{4 \pi^{2}}{a^{2}}\left(m^{2}+n^{2}\right)\left(\frac{f}{\rho_{c}}+\frac{1-f}{\rho_{f}}\right)
\end{aligned}
$$

and off-diagonal

$$
\begin{aligned}
& A\left(\mathbf{G}, \mathbf{G}^{\prime}\right)=A_{m n, m^{\prime} n^{\prime}}=\left(\frac{1}{\rho_{c}}-\frac{1}{\rho_{f}}\right) \frac{4\left(m m^{\prime}+n n^{\prime}\right)}{a^{2}\left(m-m^{\prime}\right)\left(n-n^{\prime}\right)} \times \\
& \times \sin \left[\frac{\pi b_{x}\left(m-m^{\prime}\right)}{a}\right] \sin \left[\frac{\pi b_{y}\left(n-n^{\prime}\right)}{a}\right]
\end{aligned}
$$

elements.

To calculate the elements $\rho_{x}$ and $\rho_{y}$ of the mass density tensor we substitute Eqs. (9)-(12) into Eqs. (7) and (8). The number of plane waves used in numerical calculations is $N=4726$ that guarantees less than $1 \%$ of numerical error. The result is shown in Fig. 1 for the interval of the filling fractions from zero to the maximum value of 0.2 (close packing structure). Both elements of the mass density tensor increase monotonically with filling fraction due to increase of inertia of the structure containing more metal. 


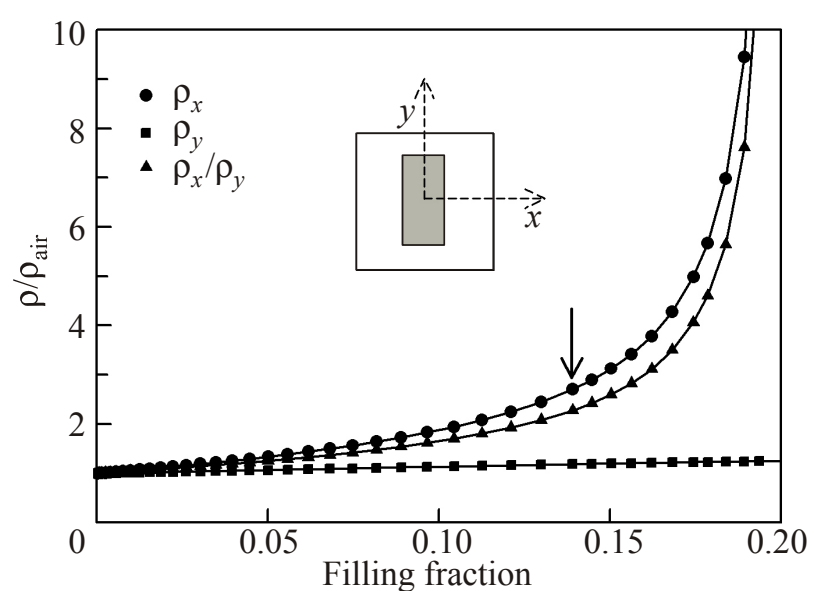

Fig. 1. Dynamical mass density (normalized to the density of air) calculated from Eqs. (7) and (8) vs filling fraction. Arrow indicates the filling fraction $f=0.14$ of the phononic crystal studied in Refs. 21 and 22. At $f=0.14$ we obtained the values $\rho_{x} / \rho_{\text {air }}=2.70$ and $\rho_{y} / \rho_{\text {air }}=1.19$ that are indistinguishable from those reported in [21] and [22]. Insert shows the unit cell of the phononic crystal.

However, the increase of the mass density along axis $x$ is much more drastical. When the spacing between the cylinders becomes very narrow, $f \rightarrow 0.2$, the mass density $\rho_{x}$ exhibits practically singular behavior. Making the spacing very narrow one can obtain a metafluid with very high level of anisotropy, $\rho_{x} / \rho_{y} \gg 1$. This increase of dynamic mass density occurs since for the narrow spacings an essential part of sound energy propagates through the metal, which has practically infinite mass density as compared to air.

\section{Conclusions}

We propose a new exact formula for the effective mass density of periodic metafluids which is valid in the long wavelength limit. The obtained result for the effective mass density of air with embedded aluminum cylinders is very close to the experimental and numerical values obtained recently in Refs. 21 and 22. Our formula is free from limitations over the geometry and the physical parameters of the periodic structure; it provides high accuracy of calculations with relatively low number of plane waves. The result of this study may be useful for design of artificial metamaterials with high level of anisotropy that are necessary for fabrications of acoustic cloaks.

This work is supported by the US Department of Energy grant \# DE-FG02-06ER46312.

1. T. Gorishny, M. Maldovan, C. Ullal, and E. Thomas, Physics World 18, 24 (2005).

2. Metamaterials: Theory, Design, and Application, Tie Jun Cui, David R. Smith, and Ruopeng Liu (eds.), Springer, NY (2009).
3. Laszlo Solymar and Ekaterina Shamonina, Waves in Metamaterials, Oxford University Press (2009).

4. Metamaterials: Critique and Alternatives, Ben A. Munk, Wiley, New Jersey (2009).

5. A.N. Norris, J. Acoust. Soc. Am. 125, 839 (2009).

6. Shu Zhang, Leilei Yin, and Nicholas Fang, Phys. Rev. Lett. 102, 194301 (2009).

7. Jensen Li, Lee Fok, Xiaobo Yin, Guy Bartal, and Xiang Zhang, Nature Materials 8, 931 (2009).

8. U. Leonhardt, Science 312, 1777 (2006); J.B. Pendry, D. Schurig, and D.R. Smith, Science 312, 1780 (2006); H. Chen, C.T. Chan, and P. Sheng, Nature Materials 9, 387 (2010).

9. Huanyang Chen and C.T. Chan, J. Phys. D: Appl. Phys. 43, 113001 (2010).

10. G.W. Milton, M. Briane, and J.R. Willis, New J. Phys. 8, 248 (2006); Formal Structure of Electromagnetics: General Covariance and Electromagnetics, E.J. Post (ed.), Wiley, New York (2006).

11. D. Schurig, J.J. Mock, B.J. Justice, S.A. Cummer, J.B. Pendry, A.F. Starr, and D.R. Smith, Science 314, 977 (2006).

12. W. Cai, U.K. Chettiar, A.V. Kildishev, and V.M. Shalaev, Nature Photonics 1, 224 (2007).

13. G.W. Milton, M. Briane, and J.R. Willis, New J. Phys. 8, 248 (2006).

14. L. Kelders, J.F. Allard, and W. Lauriks, J. Acoust. Soc. Am. 103, 2730 (1998).

15. S.A. Cummer and D. Schurig, New J. Phys. 9, 45 (2007).

16. D. Torrent and J. Sánchez-Dehesa, New J. Phys. 9, 323 (2007).

17. D. Torrent and J. Sánchez-Dehesa, New J. Phys. 10, 023004 (2008).

18. D. Torrent and J. Sánchez-Dehesa, New J. Phys. 10, 063015 (2008).

19. D. Torrent and J. Sánchez-Dehesa, Phys. Rev. Lett. 103, 064301 (2009).

20. D. Torrent and J. Sánchez-Dehesa, Phys. Rev. Lett. 105, 174301 (2010).

21. B.-I. Popa and S.A. Cummer, Phys. Rev. B80, 174303 (2009).

22. L. Zigoneanu, B.-I. Popa, A.F. Starr, and S.A. Cummer, J. Appl. Phys. 109, 054906 (2011).

23. A.W. Wood, Textbook of Sound, Macmillan, New York (1941).

24. J.G. Berryman, J. Acoust. Soc. Am. 68, 1809 (1980).

25. J.C. Maxwell-Garnett, Philos. Trans. R. Soc. 203, 385 (1904).

26. J. Mei, Z. Liu, W. Wen, and P. Sheng, Phys. Rev. Lett. 96, 024301 (2006).

27. F. Cervera, L. Sanchis, J.V. Sánchez-Perez, R. Martinez-Sala, C. Rubio, F. Meseguer, C. Lopez, D. Caballero, and J. Sánchez-Dehesa, Phys. Rev. Lett. 88, 023902 (2002); L. Sanchis, A. Hakansson, F. Cervera, and J. Sánchez-Dehesa, Phys. Rev. B67, 035422 (2003).

28. A.A. Krokhin, J. Arriaga, and L. Gumen, Phys. Rev. Lett. 91, 264302 (2003).

29. A.A. Krokhin, P. Halevi, and J. Arriaga, Phys. Rev. B65, 115208 (2002); J. Arriaga, A.A. Krokhin, and P. Halevi, Physica E17, 436 (2003). 